\title{
OCEAN: a randomized Phase III study of melflufen + dexamethasone to treat relapsed refractory multiple myeloma
}

\author{
Fredrik Schjesvold ${ }^{1}$, Pawel Robak ${ }^{2}$, Ludek Pour ${ }^{3}$, Johan Aschan ${ }^{4}$ \& Pieter Sonneveld ${ }^{5, *}$ \\ ${ }^{1}$ Oslo Myeloma Center, Oslo University Hospital \& KG Jebsen Center for B Cell Malignancies, University of Oslo, Oslo, Norway \\ ${ }^{2}$ Medical University of Lodz, Lodz, Poland \\ ${ }^{3}$ University Hospital of Masaryk, Brno, Czech Republic \\ ${ }^{4}$ Oncopeptides AB, Stockholm, Sweden \\ ${ }^{5}$ Erasmus MC Cancer Institute \& Erasmus University of Rotterdam, Rotterdam, The Netherlands \\ *Author for correspondence: p.sonneveld@erasmusmc.nl
}

\begin{abstract}
Melflufen is a novel peptide-drug conjugate that rapidly delivers a cytotoxic payload into tumor cells. It has emerged as a potential new multiple myeloma treatment, particularly for late-stage forms of the disease. Here we describe the rationale and design of OCEAN (NCT03151811), a randomized, head-tohead, superiority, open-label, global, Phase III study evaluating the efficacy and safety of melflufen + dexamethasone versus pomalidomide + dexamethasone. Eligible patients with relapsed refractory multiple myeloma have received 2-4 previous treatments and are refractory to both lenalidomide and their last treatment. Patients are excluded if they have previously received pomalidomide. The primary endpoint is progression-free survival, and key secondary endpoints include overall response rate, duration of response and overall survival.
\end{abstract}

First draft submitted: 9 January 2020; Accepted for publication: 19 February 2020; Published online: 6 March 2020

Keywords: alkylating agent • melflufen • MM • multiple myeloma • Phase III study • relapsed refractory multiple myeloma • RRMM

\section{Multiple myeloma}

Multiple myeloma (MM) is the second most common hematological malignancy after non-Hodgkin's lymphoma [1]. Approximately 32,110 new cases of MM were expected to be diagnosed in the USA in 2019, accounting for $1.8 \%$ of all new cancer cases, with approximately 12,960 deaths from the disease $[2,3]$. It occurs at a higher rate in men than in women and in people of African descent compared with other ethnicities [3].

Despite considerable improvements in the clinical outcomes for patients with MM in recent decades [4-6], MM remains incurable with a high burden of disease. As the disease progresses, patients experience a repeating pattern of remission and relapse as they cycle through therapeutic options. Each period of remission is usually shorter than the last as the tumor becomes more aggressive [7]. Clonal evolution and genomic instability within myeloma clones drive disease progression and treatment resistance [8-11]. When the disease becomes progressive on therapy, or within 60 days of the last treatment, it is classified as relapsed refractory MM (RRMM) [12]. Furthermore, as patients with MM are usually elderly (median age at diagnosis of 69 years), they often present with comorbidities such as cardiovascular disease, diabetes and pulmonary disease, which not only further impact the patient's quality of life, but also limit the choice of treatment [13,14].

In recent years, multiple new treatment options have been approved for use in MM. These include the immunomodulatory drug (IMiD) pomalidomide, the proteasome inhibitors (PIs) carfilzomib and ixazomib, monoclonal antibodies (mAbs) daratumumab and elotuzumab, and most recently, the selective inhibitor of nuclear export, selinexor $[15,16]$. Although such treatments have improved clinical outcomes, treatment of patients who are refractory to previous therapies is still challenging. A growing concern among clinicians is the increasing population of patients who are refractory to front-line treatments, including lenalidomide and daratumumab, effectively

Future Medicine 


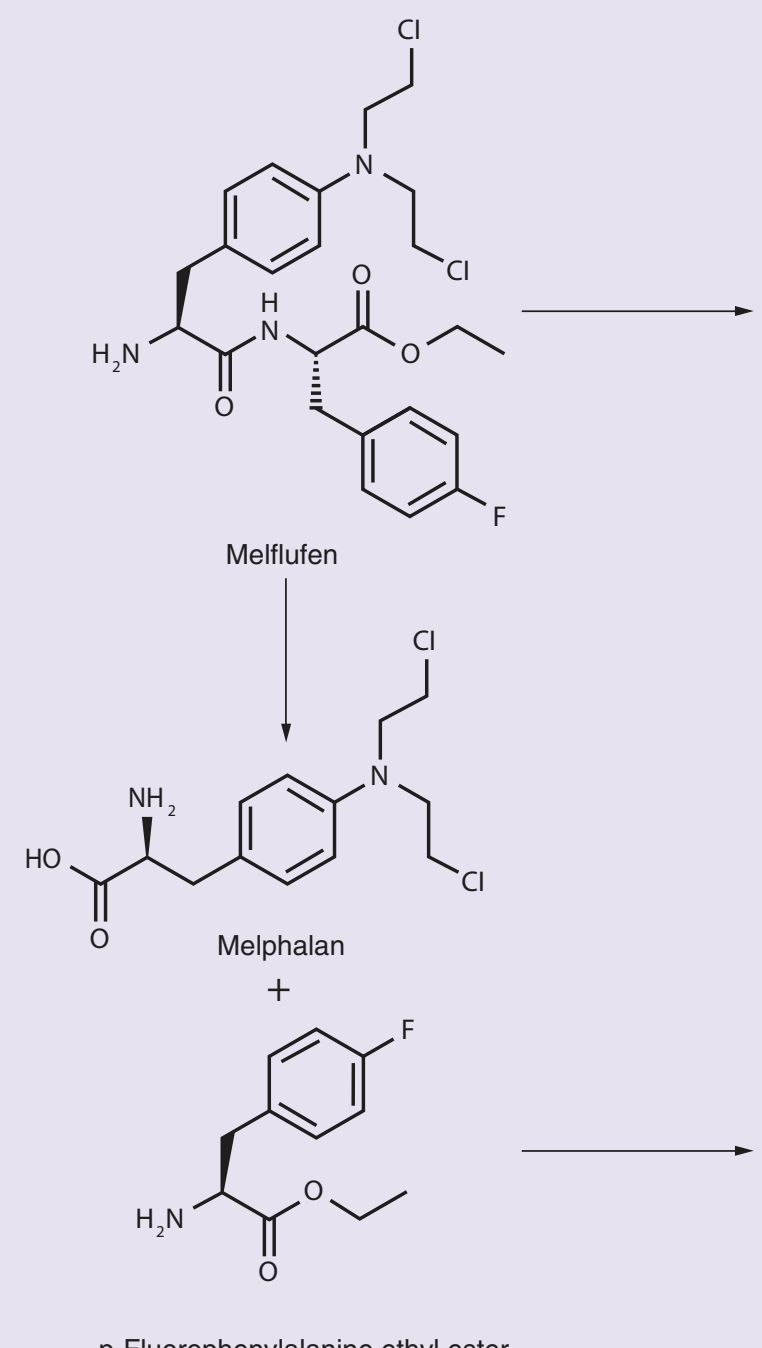<smiles>N[C@@H](Cc1ccc(N(CCCl)CCCl)cc1)C(=O)N[C@@H](Cc1ccc(F)cc1)C(=O)O</smiles>

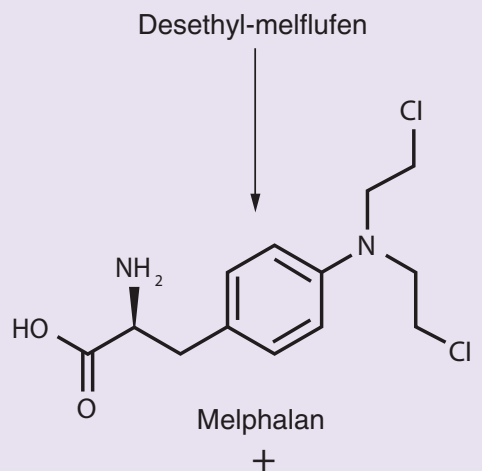

p-Fluorophenylalanine ethyl ester<smiles>N[C@@H](Cc1ccc(F)cc1)C(=O)O</smiles>

p-Fluorophenylalanine

Figure 1. The metabolism of melflufen. Melflufen (top left) is metabolized into melphalan (middle) and p-Fluorophenylalanine (bottom) by aminopeptidases inside myeloma cells.

eliminating these drugs from further use. Also, patients with RRMM are highly heterogenous; therefore, the need remains for new, efficacious drugs with novel mechanisms of action.

\section{Melflufen}

Melflufen (melphalan flufenamide) is a novel peptide-drug conjugate that rapidly delivers a cytotoxic payload into tumor cells [17]. Melflufen is highly lipophilic, allowing it to be rapidly taken up by myeloma cells [17]. The antineoplastic activity of melflufen is dependent upon the expression of aminopeptidases, like APN (also known as CD13), which cleave melflufen into melphalan and p-Fluorophenylalanine (Figure 1) [18,19]. Following aminopeptidase-dependent cleavage, the hydrophilic alkylating metabolite melphalan accumulates in myeloma cells. This enrichment of the cytotoxic payload has a substantial impact on the antimyeloma activity of melflufen $[18,20]$. Multiple cell culture studies of melflufen have demonstrated higher cytotoxicity in myeloma cells compared with peripheral blood mononuclear cells, with significant activity observed in melphalan-resistant myeloma cells [19,21,22]. Indeed, melflufen-induced apoptosis is initiated after $2 \mathrm{~h}$ of exposure to melflufen, whereas melphalan-induced apoptosis is initiated after $6 \mathrm{~h}$ of exposure [21]. This rapid cytotoxicity may explain how melflufen can overcome melphalan-resistance in vitro and in xenograft models. In patient-derived myeloma tumor samples, melflufen 
MELFLUFEN DEVELOPMENT PROGRAM IN MULTIPLE MYELOMA
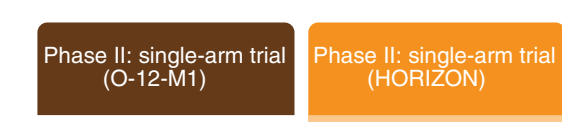

Show single-agent activity in RRMM

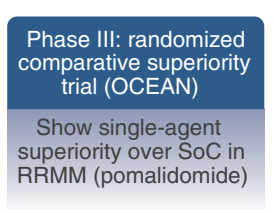

Phase III: randomized

Show single-agent superiority over SoC in
RRMM (pomalidomide)
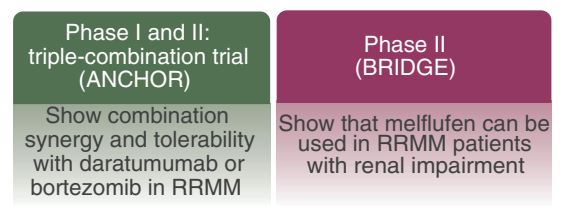

(O-12-M1)

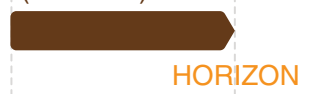

OCEAN

2016

2017

2018

2019

2020

2021

Figure 2. The clinical development of melflufen.

Reproduced with the permission of the owner, Oncopeptides $A B \odot$.

RRMM: relapsed refractory multiple myeloma; SoC: standard of care.

demonstrated approximately 50-fold higher cytotoxicity than melphalan, and exposure of melphalan was 50-fold higher with melflufen versus direct administration of melphalan in culture [20,21]. Furthermore, melflufen shows strong anti-angiogenic properties [22,23]. Finally, the first-in-human study of intravenous melflufen in solid tumors showed that it could be administered safely at active doses with a hematologic toxicity profile as expected for an alkylating agent [24].

\section{Clinical development of melflufen}

Melflufen has been in clinical development in multiple myeloma since 2013, as outlined here. The OCEAN study detailed in this report builds on encouraging results from two prior studies (Figure 2): a completed Phase I/II study (O-12-M1, NCT01897714) and an ongoing Phase II study (HORIZON, NCT02963493). Two additional Melflufen trials have been initiated since the OCEAN study began and are currently ongoing (ANCHOR, NCT03481556 and BRIDGE, NCT03639610; Figure 2). Finally, a Phase I/II open-label dose-escalation and dose-expansion study of melflufen in combination with dexamethasone in patients with immunoglobulin lightchain (AL) amyloidosis following at least one prior line of therapy is also planned to begin in 2020 (OP-102, NCT04115956). The plasma cell dyscrasia associated with AL amyloidosis resembles that observed in MM; therefore, therapies that are effective in MM are often used to treat AL amyloidosis. The primary endpoint of the Phase II part of this study will be to determine overall response rate (ORR).

The Phase I part of the O-12-M1 study determined the maximum tolerated dose of melflufen as $40 \mathrm{mg}$ every 21 days in combination with $40 \mathrm{mg}$ dexamethasone weekly [25]; the optimal cycle length was increased from 21 to 28 days following a Phase II protocol amendment. The Phase II part assessed the activity of melflufen in 45 patients with late-stage RRMM who had received a median of four prior lines of treatment and who were refractory to both lenalidomide (an IMiD) and bortezomib (a PI). Melflufen + dexamethasone treatment resulted in disease stabilization in the majority of patients, with a favorable median time to next treatment and ORR [26]. Furthermore, the clinical response observed in this study was durable, which is promising given the advanced nature 
of the disease in these patients [26]. The safety profile of melflufen in the O-12-M1 study demonstrated predictable and manageable adverse events (AEs), with the most common being grade 3 and 4 hematological AEs and low rates of non-hematological AEs [26].

In addition to the positive results from the O-12-M1 study, the ongoing Phase II HORIZON study is assessing the efficacy and safety of melflufen + dexamethasone in 157 patients with RRMM [27]. These patients are refractory to pomalidomide and/or daratumumab, two of the most recently approved treatments for RRMM. Preliminary assessment of the available data (data cutoff: 6th May 2019, $\mathrm{n}=121$ ) showed disease stabilization in the majority of patients, with promising ORRs in both double- and triple-class refractory patients (including patients refractory to the current standard of care, pomalidomide) [28]. The safety profile was comparable to that observed in the O-12-M1 study.

Thus, the Phase I/II O-12-M1 and HORIZON studies provided preliminary evidence that melflufen is effective in heavily pretreated patients who are refractory to various currently available therapies, having few remaining treatment alternatives. Building on these findings, the rationale for the Phase III OCEAN study is to compare the efficacy of melflufen to that of the current standard of care for these patients, pomalidomide. If melflufen is shown to provide clinical benefit beyond that of pomalidomide, this may lead to the approval of melflufen for use in patients in the real-world setting.

\section{The OCEAN study}

\section{Introduction}

OCEAN (NCT03151811) is a randomized, head-to-head, superiority, open-label, global, Phase III study evaluating the efficacy and safety of melflufen + dexamethasone versus pomalidomide + dexamethasone in patients with MM who have received 2-4 prior therapies; patients are refractory to both their last line of therapy and lenalidomide within 18 months of randomization. This study is sponsored by Oncopeptides AB.

\section{Background \& study rationale}

As described in detail below, enrollment in the OCEAN study required that patients with RRMM were not primary refractory, had received at least 2-4 lines of prior therapy, and were refractory to both lenalidomide and the last line of treatment. These patients have very few remaining treatment options and are in need of novel treatments to improve their clinical outcomes.

Pomalidomide, a derivative of the IMiD thalidomide, is the current standard of care for patients with RRMM who are refractory to both lenalidomide and bortezomib [29,30]. The approval of pomalidomide was based on the Phase III study MM-003 (NIMBUS, NCT01311687), which showed that pomalidomide in combination with low-dose dexamethasone is superior to combination with high-dose dexamethasone (median PFS of 4.0 vs 1.9 months; $\mathrm{p}<0.0001$ ) [31]. Many features of the OCEAN study design, such as patient population and dose regimen, resemble those of the MM-003 study; this is to ensure that comparisons between the two studies are as robust as possible.

The institutional review board or independent ethics committee of each investigational site approved the OCEAN protocol. The study is being performed in accordance with the Declaration of Helsinki and Good Clinical Practice.

Study design

Eligibility criteria

Eligible patients (males and females aged $\geq 18$ years) with RRMM must have measurable disease and received 2-4 prior lines of therapy, including lenalidomide and a PI. Patients must be refractory to both lenalidomide and the last line of therapy, and have adequate hematologic and organ function. Patients will be excluded if they have primary refractory disease, previously received pomalidomide, or have select comorbid conditions or residual side effects from prior therapy above grade 1. Key eligibility criteria can be found in Box 1.

\section{Screening}

All patients must provide written informed consent before any protocol-specific screening tests or procedures are performed. Screening is performed between days -21 and -1 , when patient eligibility, baseline characteristics, clinical assessment and disease evaluation are assessed. Of note, renal function is assessed both during the screening process and immediately prior to study drug administration (Cycle 1, Day 1). An estimated creatine clearance rate by Cockcroft-Gault formula of $>45 \mathrm{ml} / \mathrm{min}$ is required for continuation with the study. The safety and 


\section{Key inclusion criteria}

- Male or female, aged 18 years or older.

- Prior diagnosis of MM with documented disease progression in need of treatment at time of screening.

- Measurable disease defined as any of the following:

- Serum M-protein $\geq 0.5 \mathrm{~g} / \mathrm{dl}$ by SPEP.

- Urine M-protein $\geq 200 \mathrm{mg} / 24 \mathrm{~h}$ by 24 -h UPEP.

- SFLC $\geq 10 \mathrm{mg} / \mathrm{dl}$ and abnormal serum kappa-to-lambda free light chain ratio (SFLC ratio).

- Received 2-4 prior lines of therapy, including lenalidomide and a PI either sequentially or in the same line, and refractory to both the last line of therapy and lenalidomide $(\geq 10 \mathrm{mg})$ administered within 18 months prior to randomization.

- Life expectancy of $\geq 6$ months and an Eastern Cooperative Oncology Group performance status $\leq 2$.

- A 12-lead ECG with QTcF interval of $<470$ ms.

- The following laboratory results must be met during screening (within 21 days) and immediately before study drug administration on Cycle 1, Day 1:

- Absolute neutrophil count $\geq 1000$ cells $/ \mathrm{mm}^{3}\left(1.0 \times 10^{9} / \mathrm{l}\right)$.

- Platelet count $\geq 75,000$ cells $/ \mathrm{mm}^{3}\left(75 \times 10^{9} / \mathrm{l}\right)$.

- Hemoglobin $\geq 8.0 \mathrm{~g} / \mathrm{dl}$.

- Total bilirubin $\leq 1.5 \times$ ULN.

- AST and ALT $\leq 3.0 \times$ ULN.

- Renal function: estimated creatinine clearance by Cockcroft-Gault formula $\geq 45 \mathrm{ml} / \mathrm{min}$.

\section{Key exclusion criteria}

- Primary refractory disease (i.e. never had at least minimal response to any prior therapy).

- Evidence of mucosal or internal bleeding and/or platelet transfusion refractory.

- Any medical conditions that, in the investigator's opinion, would impose excessive risk to the patient or adversely affect participation in the study.

- Prior exposure to pomalidomide.

- Known intolerance to IMiDs (grade $\geq 3$ hypersensitivity reaction or at the investigator's discretion).

- Known active infection requiring parenteral or oral anti-infective treatment within 14 days of randomization.

- Other malignancy diagnosed or requiring treatment within the past 3 years, with the exception of adequately treated basal cell carcinoma, squamous cell skin cancer, carcinoma in situ of the cervix or breast and very low- or low-risk prostate cancer under active surveillance.

- Concurrent symptomatic amyloidosis or plasma cell leukemia.

ECG: Eectrocardiogram; IMiD: Immunomodulatory imide drug; QTCF: QT interval calculated by Fredericia Formula; SFLC: Serum-free light chains; SPEP: Serum protein electrophoresis; ULN: Upper limit of normal; UPEP: Urine protein electrophoresis.

pharmacokinetics of melflufen in patients with renal impairment is being assessed in the ongoing BRIDGE trial (NCT03639610).

The cytogenetics of the patient population will be assessed by fluorescence in situ hybridization (FISH) during screening. This will provide insight into the frequency of cytogenetic abnormalities within the study population and may inform on the efficacy of melflufen in patients with varying degrees of cytogenetic risk.

Patients are stratified using three criteria: age ( $<75$ vs $\geq 75$ years); number of prior lines of treatment ( 2 vs $3-4$ ); and International Staging System score $(1 \mathrm{vs} \geq 2)$ [32]. Prior to randomization, confirmation of patient eligibility by the medical monitor is required. Randomization is managed by interactive response technology software and places each patient into one of two study arms.

\section{Treatment}

Treatment is required to begin within 5 days of randomization. Each treatment cycle lasts 28 days. Patients will only stop receiving treatment if: i) there is documented disease progression; ii) there is unacceptable toxicity; or iii) the patient or treating physician determines that it is not in the patient's best interest to continue.

The treatment doses and schedule are summarized in Table 1. Patients randomized to arm A will receive $40 \mathrm{mg}$ melflufen intravenously on Day 1 and $40 \mathrm{mg}$ dexamethasone orally (tablets) on days 1, 8, 15 and 22 of each 28-day cycle. Patients randomized to arm B will receive $4 \mathrm{mg}$ pomalidomide capsules orally on days 1-21 and $40 \mathrm{mg}$ dexamethasone orally (tablets) on days 1, 8, 15 and 22 of each 28 -day cycle. For patients aged $\geq 75$ years, the dexamethasone dose in both arms will be reduced from $40 \mathrm{mg}$ to $20 \mathrm{mg}$ on days 1, 8, 15 and 22 of each 28-day cycle. 
Table 1. OCEAN study dose schedule.

\begin{tabular}{|c|c|c|c|}
\hline Group & Drug & Dose & Schedule (/28-day cycle) \\
\hline \multirow[t]{2}{*}{ Arm A } & Melflufen & $40 \mathrm{mg} \mathrm{IV}$ & Day 1 \\
\hline & Dexamethasone & $40 \mathrm{mg}$ oral tablets ${ }^{\dagger}$ & Days $1,8,15$ and 22 \\
\hline \multirow[t]{2}{*}{ Arm B } & Pomalidomide & $4 \mathrm{mg}$ oral capsule & Days 1-21 (inclusive) \\
\hline & Dexamethasone & $40 \mathrm{mg}$ oral tablets ${ }^{\dagger}$ & Days $1,8,15$ and 22 \\
\hline
\end{tabular}

†The dexamethasone dose will be reduced to $20 \mathrm{mg}$ for patients aged $\geq 75$ years. In the USA only, oral dexamethasone may be substituted with IV dexamethasone at the investigator's discretion.

IV: Intravenous.

\section{Table 2. Required and recommended concomitant therapy.}

\begin{tabular}{|c|c|c|}
\hline Group(s) & Concomitant therapy & Comment \\
\hline \multicolumn{3}{|c|}{ Required therapy } \\
\hline Both arms & Contraception & $\begin{array}{l}\text { All patients of child-bearing potential from both treatment arms are required to use two } \\
\text { effective methods of contraception (or true abstinence, i.e. in line with the preferred and } \\
\text { usual lifestyle of the patient) prior to randomization. Following randomization, contraception } \\
\text { should be used as per protocol }\end{array}$ \\
\hline Arm B & Anti-thrombotic prophylactic treatment & $\begin{array}{l}\text { Pomalidomide treatment increases the risk of thromboembolism. Unless otherwise indicated, } \\
\text { patients should receive prophylactic anti-thrombotic treatment }\end{array}$ \\
\hline \multicolumn{3}{|c|}{ Recommended therapy } \\
\hline Both arms & Pneumocystic prophylaxis & $\begin{array}{l}\text { All patients are recommended to receive pneumocystis prophylaxis according to the NCCN or } \\
\text { institutional guidelines }\end{array}$ \\
\hline $\operatorname{Arm~A}$ & Anti-emetic propylaxis & $\begin{array}{l}\text { Anti-emetic prophylaxis prior to melflufen administration is recommended. Subsequent } \\
\text { anti-emetic treatment is at the investigator's discretion }\end{array}$ \\
\hline Both arms & $\begin{array}{l}\text { Full supportive care for tumor lysis } \\
\text { syndrome }\end{array}$ & $\begin{array}{l}\text { To include transfusions of blood and blood products (including platelets), antibiotics, } \\
\text { antidiarrheals, analgesics etc. and prophylactic treatment for tumor lysis syndrome when } \\
\text { appropriate }\end{array}$ \\
\hline Both arms & Bisphosphonate therapy & To be administered if indicated by institutional guidelines \\
\hline Both arms & $\begin{array}{l}\text { Supportive measures for } \\
\text { thrombocytopenia and neutropenia }\end{array}$ & $\begin{array}{l}\text { General supportive measures, together with appropriate blood and platelet transfusions and } \\
\text { hematological growth factors should be administered if necessary. It is recommended, at the } \\
\text { investigator's discretion, that platelet transfusion should be avoided within } \leq 5 \text { days of the } \\
\text { next dose of study treatment (except Cycle 1, Day } 1 \text { ). This is to assess endogenous platelet } \\
\text { recovery and to avoid excessive myelosuppression. Careful attention is to be paid to } \\
\text { monitoring of blood counts }\end{array}$ \\
\hline Both arms & Anti-microbial prophylaxis & $\begin{array}{l}\text { Recommended for patients with a history of CMV infection and those with neutropenia, } \\
\text { according to the NCCN or institutional guidelines. Other anti-viral and anti-fungal treatments } \\
\text { are to be considered, as required }\end{array}$ \\
\hline
\end{tabular}

All required and recommended concomitant therapies for the OCEAN study are summarized in Table 2.

\section{Follow-up}

Both PFS follow-up and OS follow-up assessments should be performed. For patients who discontinue treatment for reasons other than progression, PFS follow-up assessments will be performed. OS follow-up assessments will be performed for all patients. Patients will only be exempt from these follow-ups in cases of death, loss to follow-up or specific withdrawal of consent for follow-up.

\section{Primary \& secondary objectives}

The primary objective of the OCEAN study is to compare the PFS of melflufen + dexamethasone and pomalidomide + dexamethasone, which will be assessed by the independent review committee using the IMWG consensus response criteria [12]. Key secondary objectives include comparison of ORR, duration of response (DOR), OS, and safety and tolerability between patients receiving melflufen + dexamethasone and those receiving pomalidomide + dexamethasone.

\section{Planned study period}

The first participant was randomized into the OCEAN study on 12 June 2017. The study has an estimated completion date of Q1 2022, with the last patient in (LPI) estimated to be Q1 2020. 


\section{Study procedures}

Efficacy evaluations

In the OCEAN study, disease assessment will be performed during screening, on Cycle 1 Day 1 , and on the planned Day 1 of each cycle, even if treatment is delayed. The amount of M-protein will be determined by SPEP, UPEP or assessment of dFLC and SFLC ratio. Immunofixation electrophoresis of both blood and urine samples will be used to determine the type of M-protein. For patients with immunoglobulin A ( $\operatorname{IgA})$ or $\operatorname{IgD}$ myeloma, quantitative $\operatorname{Ig}$ testing will also be performed because of the difficulty in assessing these disease types with SPEP [33,34]. Assessment of dFLC and SFLC ratio is not necessary if there is measurable SPEP or UPEP but SFLC ratio is required to confirm a stringent complete response (sCR), as defined by the IMWG criteria for diagnosis of MM [35].

In addition to M-protein assessment, disease status will be determined using skeletal $\mathrm{x}$-rays and/or computed tomography scans of bones, bone marrow aspirate, and imaging procedures of known or suspected extramedullary plasmacytomas. These assessments, in addition to serum calcium assessment, will then be performed throughout the study according to IMWG response criteria [11].

\section{Safety \& tolerability evaluations}

AEs will be assessed and graded according to Common Terminology Criteria for Adverse Events (v4.03). Other safety and tolerability assessments will include physical assessments (vital signs, neurological assessment and performance status assessment), routine safety laboratory tests, chest $\mathrm{x}$-rays, hepatitis B screens, pregnancy testing and electrocardiograms. An independent data safety monitoring committee will regularly evaluate the risk-benefit ratio for enrolled patients.

\section{Pharmacokinetic evaluations}

Melphalan concentrations will be determined from plasma samples for the first two treatment cycles of melflufen. Samples will be taken at three time points following infusion: 10-15 min, $1 \mathrm{~h}$ and $2-4 \mathrm{~h}$ (as late as possible within the time frame).

\section{Statistical analysis}

Patients are being enrolled at approximately 140 sites in 21 countries. The sample size calculation was based on the primary objective of comparing PFS between the two arms of the study. In order to reach the target of 339 events within 6 months after randomization of the last patient, it is anticipated that a total of 450 patients will need to be randomized over 24 months; this is assuming a median PFS for the control arm (arm B) of 3.6 months [30]. This design is based on a two-sided significance level of $0.05 \%$ and has a power of $90 \%$ to detect a hazard ratio of 0.70 .

PFS is defined as the time (in months) from the date of randomization to the earlier of either confirmed disease progression or death from any cause. It will be analysed by applying a two-sided, 0.05-level stratified Cox proportional hazards regression model on the full analysis set (defined as all randomized patients). Superiority of melflufen + dexamethasone will be demonstrated if the upper limit of the $95 \%$ confidence interval (CI) for median PFS is below 1.0, while non-inferiority will be demonstrated if the upper limit is below 1.2. Superiority and non-inferiority assessments are both included because the US FDA and some EU authorities have different requirements when assessing approval for new therapies.

ORR is defined as a best confirmed response of sCR, complete response (CR), very good partial response (VGPR) or PR using local laboratory evaluation, according to IMWG guidelines [12]. The ORR and approximate $95 \%$ CI will be determined for each arm of the study using the Cochran-Mantel-Haenszel $\chi^{2}$ test. DOR is defined as the time (in months) from the first evidence of confirmed assessment of sCR, CR, VGPR or PR to first confirmed disease progression or death from any cause. DOR is defined only for patients with a confirmed PR or better. OS is defined as the time (in months) from the date of randomization to death from any cause. DOR and OS will be analysed as described for PFS. No formal statistical analysis will be performed on the safety endpoints.

\section{Conclusion}

The OCEAN study will evaluate whether melflufen is superior to pomalidomide as combination therapy with dexamethasone for the treatment of advanced RRMM. It is hoped that this Phase III study, in conjunction with other clinical studies of melflufen, will result in the availability of a new treatment option that will improve clinical outcomes for patients with late-stage RRMM. 
Executive summary

Background to multiple myeloma

- Multiple myeloma (MM) is an incurable clonal B-cell malignancy of the plasma cells in bone marrow.

- Owing to clonal heterogeneity and evolution of myeloma cells, MM ultimately progresses to refractory relapsed MM (RRMM). Patients with RRMM will typically experience diminishing periods of remission as a result of treatment resistance.

- There is an unmet medical need for new treatments against the most aggressive and late-stage forms of RRMM.

Melflufen

- Melflufen is a novel peptide-drug conjugate that rapidly delivers a cytotoxic payload into tumor cells.

- The mechanism of action of melflufen results in the delivery of a rapid and high dose of a cytotoxic alkylator payload to myeloma cells, attenuating the development of treatment resistance.

- The completed Phase I/II O-12-M1 study investigated the use of melflufen in patients with RRMM refractory to both lenalidomide and bortezomib (objective response rate [ORR] 31\%, duration of response 8.4 months, progression-free survival [PFS] 5.7 months, overall survival [OS] 20.7 months).

- The ongoing Phase II HORIZON study is investigating the use of melflufen in patients with RRMM refractory to pomalidomide and/or daratumumab (ORR 28\%, PFS 4 months, OS 11.2 months [8.5 months in patients with triple-refractory disease]).

The OCEAN study

- OCEAN is a randomized, head-to-head, superiority, open-label, global, Phase III study evaluating the efficacy and safety of melflufen + dexamethasone versus pomalidomide + dexamethasone.

- Eligible patients are refractory to both lenalidomide and the last line of therapy, and must not have previously received pomalidomide.

- Patients randomized to arm A will receive $40 \mathrm{mg}$ melflufen intravenously on Day 1 of every cycle and $40 \mathrm{mg}$ oral dexamethasone on days 1, 8, 15 and 22 of each cycle. Patients randomized to arm B will receive $4 \mathrm{mg}$ pomalidomide capsule daily on days 1-21 of each cycle and $40 \mathrm{mg}$ oral dexamethasone tablet on days $1,8,15$ and 22 of each cycle.

- The primary endpoint is PFS.

- Secondary endpoints include OS, duration of response, ORR and safety.

Conclusion

- It is hoped that the OCEAN study will demonstrate melflufen to be superior to pomalidomide as combination therapy with dexamethasone in patients with late-stage RRMM.

- If proved, this may lead to the availability of melflufen as a new treatment option to improve the clinical outcomes of patients with advanced RRMM.

Author contributions

All authors contributed equally to the manuscript and all met the following criteria:

- Substantial contributions to the conception or design of the work; or the acquisition, analysis, or interpretation of data for the work;

- Drafting the work or revising it critically for important intellectual content;

- Final approval of the version to be published;

- Agreement to be accountable for all aspects of the work in ensuring that questions related to the accuracy or integrity of any part of the work are appropriately investigated and resolved.

Financial \& competing interests disclosure

The OCEAN study was funded by Oncopeptides AB. F Schjesvold has participated in advisory boards for Oncopeptides AB. J Aschan is a Medical Director at Oncopeptides AB. P Sonneveld has participated in advisory boards for Oncopeptides AB and has participated in advisory boards and provided research support for Celgene. The authors have no other relevant affiliations or financial involvement with any organization or entity with a financial interest in or financial conflict with the subject matter or materials discussed in the manuscript apart from those disclosed.

Medical writing support was provided by A Smith, PhD, from Mudskipper Business Limited, funded by Oncopeptides AB.

Ethical conduct of research

The authors state that they have obtained appropriate institutional review board approval or have followed the principles outlined in the Declaration of Helsinki for all human or animal experimental investigations. In addition, for investigations involving human subjects, informed consent has been obtained from the participants involved. 


\section{Open access}

This work is licensed under the Attribution-NonCommercial-NoDerivatives 4.0 Unported License. To view a copy of this license, visit http://creativecommons.org/licenses/by-nc-nd/4.0/

\section{References}

Papers of special note have been highlighted as: $\bullet$ of interest; $\bullet \bullet$ of considerable interest

1. Ramsenthaler C, Osborne TR, Gao W et al. The impact of disease-related symptoms and palliative care concerns on health-related quality of life in multiple myeloma: a multi-centre study. BMC Cancer 16, 427 (2016).

2. Siegel RL, Miller KD, Jemal A. Cancer statistics, 2016. CA Cancer J. Clin. 66(1), 7-30 (2016).

3. SEER. Surveillance, epidemiology, and end results program cancer statistics stat fact sheet:myeloma. National Cancer Institute (2019). https://seer.cancer.gov/statfacts/html/mulmy.html

4. Kvam AK, Waage A. Health-related quality of life in patients with multiple myeloma - does it matter? Haematologica 100(6), 704-705 (2015).

5. Ramsenthaler C, Kane P, Gao W et al. Prevalence of symptoms in patients with multiple myeloma: a systematic review and meta-analysis. Eur. J. Haematol. 97(5), 416-429 (2016).

6. Moore HE, Davenport EL, Smith EM et al. Aminopeptidase inhibition as a targeted treatment strategy in myeloma. Mol. Cancer Ther. 8(4), 762-770 (2009).

7. Yong K, Delforge M, Driessen C et al. Multiple myeloma: patient outcomes in real-world practice. Br. J. Haematol. 175(2), 252-264 (2016).

8. Kumar SK, Dimopoulos MA, Kastritis E et al. Natural history of relapsed myeloma, refractory to immunomodulatory drugs and proteasome inhibitors: a multicenter IMWG study. Leukemia 31(11), 2443-2448 (2017).

9. Tamura H. Immunopathogenesis and immunotherapy of multiple myeloma. Int. J. Hematol. 107(3), 278-285 (2018).

10. Brioli A, Melchor L, Cavo M, Morgan GJ. The impact of intra-clonal heterogeneity on the treatment of multiple myeloma. Br. J. Haematol. 165(4), 441-454 (2014).

11. Keats JJ, Chesi M, Egan JB et al. Clonal competition with alternating dominance in multiple myeloma. Blood 120(5), 1067-1076 (2012).

12. Rajkumar SV, Harousseau J-L, Durie B et al. Consensus recommendations for the uniform reporting of clinical trials: report of the International Myeloma Workshop Consensus Panel 1. Blood 117(18), (2011).

13. Kumar SK, Rajkumar V, Kyle RA et al. Multiple myeloma. Nat. Rev. Dis. Primers 3, 17046 (2017).

14. Moreau P, San Miguel J, Sonneveld P et al. Multiple myeloma: ESMO Clinical Practice Guidelines for diagnosis, treatment and follow-up. Ann. Oncol. 28(Suppl. 4), iv52-iv61 (2017).

15. Raza S, Safyan RA, Rosenbaum E, Bowman AS, Lentzsch S. Optimizing current and emerging therapies in multiple myeloma: a guide for the hematologist. Ther. Adv. Hematol. 8(2), 55-70 (2017).

16. Chari A, Vogl DT, Gavriatopoulou M et al. Oral selinexor-dexamethasone for triple-class refractory multiple myeloma. New Engl. J. Med. 381(8), 727-738 (2019).

17. Wickström M, Nygren P, Larsson R et al. Melflufen - a peptidase-potentiated alkylating agent in clinical trials. Oncotarget 8(39), 6641 (2017).

18. Gullbo J, Wickstrom M, Tullberg M et al. Activity of hydrolytic enzymes in tumour cells is a determinant for anti-tumour efficacy of the melphalan containing prodrug J1. J. Drug Target. 11(6), 355-363 (2003).

19. Wickström M, Viktorsson K, Lundholm L et al. The alkylating prodrug J1 can be activated by aminopeptidase N, leading to a possible target directed release of melphalan. Biochem. Pharmacol. 79(9), 1281-1290 (2010).

-. Identifies the role of aminopeptidase $\mathrm{N}$ in cleavage of melflufen and the targeted release of the alkylating payload, melphalan, in myeloma cells.

20. Chauhan D, Ray A, Viktorsson $\mathrm{K}$ et al. In vitro and in vivo antitumor activity of a novel alkylating agent, melphalan-flufenamide, against multiple myeloma cells. Clin. Cancer Res. 19(11), 3019-3031 (2013).

21. Ray A, Ravillah D, Das DS et al. A novel alkylating agent Melflufen induces irreversible DNA damage and cytotoxicity in multiple myeloma cells. Br. J. Haematol. 174(3), 397-409 (2016).

- Investigates melflufen-induced apoptosis in melphalan-resistant myeloma cells.

22. Wickström M, Haglund C, Lindman H, Nygren P, Larsson R, Gullbo J. The novel alkylating prodrug J1: diagnosis directed activity profile ex vivo and combination analyses in vitro. Invest. New Drugs 26(3), 195-204 (2008).

23. Strese $\mathrm{S}$, Wickstrom M, Fuchs PF et al. The novel alkylating prodrug melflufen (J1) inhibits angiogenesis in vitro and in vivo. Biochem. Pharmacol. 86(7), 888-895 (2013). 
24. Berglund A, Ullen A, Lisyanskaya A et al. First-in-human, Phase I/IIa clinical study of the peptidase potentiated alkylator melflufen administered every three weeks to patients with advanced solid tumor malignancies. Invest. New Drugs 33(6), 1232-1241 (2015).

25. Paba-Prada C, Palumbo A, Mellqvist U-H et al. Determination of the MTD and encouraging results in an ongoing open-label Phase $1 / 2$ a study of the safety and efficacy of melflufen and dexamethasone in combination for patients with relapsed and relapsed-refractory multiple myeloma (RRMM). Blood 124(21), 2123 (2014).

26. Bringhen S, Richardson PG, Vorhees P et al. O-12-M1: an evaluation of time to next treatment in melflufen and dexamethasone-treated patients with relapsed/refractory multiple myeloma (Poster PF628). 24th European Hematology Association Annual Meeting, Amsterdam, The Netherlands (2019).

-• Completed supporting Phase I/II trial assessing clinical benefit of melflufen in 45 patients with RRMM.

27. NIH Clinical Trials. A study of melphalan flufenamide (melflufen) in combination with dexamethasone in relapsed refractory multiple myeloma patients (HORIZON) (2020). www.clinicaltrials.gov/ct2/show/NCT02963493?term=melflufen+horizon\&draw=2\&rank=1 - Ongoing pivotal Phase II trial assessing overall response rate in 157 patients with RRMM treated with melflufen.

28. Richardson PG, Oriol A, Larocca A et al. HORIZON (OP-106): updated efficacy and safety of Melflufen in relapsed/refractory multiple myeloma refractory to daratumumab and/or pomalidomide (Oral Presentation S1605). 24th European Hematology Association Annual Meeting. Amsterdam, The Netherlands (2019).

29. Rychak E, Mendy D, Shi T et al. Pomalidomide in combination with dexamethasone results in synergistic anti-tumour responses in pre-clinical models of lenalidomide-resistant multiple myeloma. Br. J. Haematol. 172(6), 889-901 (2016).

30. US FDA. FDA Pomalyst prescribing information (2015). www.accessdata.fda.gov/drugsatfda_docs/label/2013/204026lbl.pdf

31. Miguel JS, Weisel K, Moreau P et al. Pomalidomide plus low-dose dexamethasone versus high-dose dexamethasone alone for patients with relapsed and refractory multiple myeloma (MM-003): a randomised, open-label, Phase 3 trial. Lancet Oncol. 14(11), 1055-1066 (2013).

32. Palumbo A, Avet-Loiseau H, Oliva $S$ et al. Revised international staging system for multiple myeloma: a report from international myeloma working group. J. Clin. Oncol. 33(26), 2863-2869 (2015).

33. Selene II, Jose JA, Khalil MJ, Faisal MS, Malik MN. Presentation patterns, diagnostic markers, management strategies, and outcomes of IgD multiple myeloma: a systematic review of literature. Cureus 11(2), e4011 (2019).

34. Katzmann JA, Willrich MaV, Kohlhagen MC et al. Monitoring IgA multiple myeloma: immunoglobulin heavy/light chain assays. Clin. Chem. 61(2), 360-367 (2015).

35. Rajkumar SV, Dimopoulos MA, Palumbo A et al. International Myeloma Working Group updated criteria for the diagnosis of multiple myeloma. Lancet Oncol. 15(12), e538-e548 (2014). 

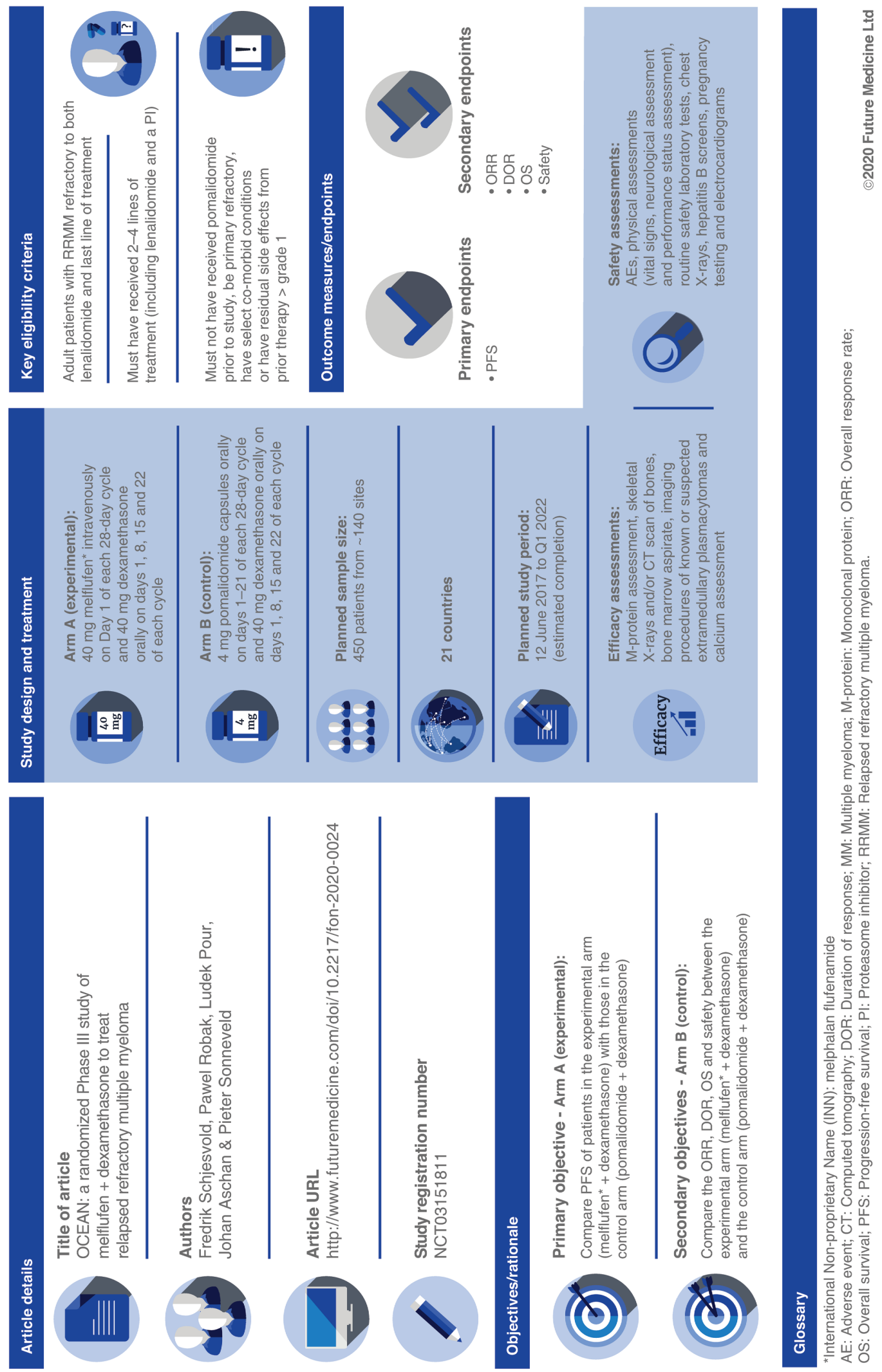